\section{P302 TRYPTOPHAN METABOLITES ARE NOT ASSOCIATED WITH DEPRESSIVE SYMPTOMS IN HIV}

T Nguyen*, J Alsop, V El-Hayek, E Cho, A Avery, C Hileman. MetrHealth Medical Center, Cleveland, USA

10.1136/sextrans-2021-sti.360

Background This study aims to determine the relationship amongst tryptophan metabolites, inflammation markers, and depression in people with HIV (PWH).

Methods This is a 12-month prospective study of chronically depressed (PHQ9 $\geq 10$ for $2+$ years) $\mathrm{PWH}$ on antiretroviral therapy (ART) to evaluate the effect of baseline plasma tryptophan (TRP), kynurenine (KYN), kynurenic acid (KA) and quinolinic acid (QA) and inflammation markers (hsCRP, IL6, sTNF-RI and -RII) quantified by LC/MS/MS and ELISA, respectively, on depressive symptoms over time. Age/sex/CD4count matched PWH with $\mathrm{PHQ} 9<5$ underwent a single visit for cross-sectional comparisons. Multivariable linear and logistic regression and linear mixed effects models were utilized.

Results 95 adults were enrolled (48 depressed; 47 control). Median age was 46; 63\% men, 35\% woman; 2\% transgender MTF; 58\% black; 36\% white; and 6\% Hispanic regardless of race. Depressed were less likely to have completed high school/GED (70 vs 91\%), were more sedentary (67 vs $18 \%$ $<10 \mathrm{hrs}$ exercise/week) and more likely to smoke (75 vs $19 \%$ ) (all $\mathrm{p}<0.03$ ). Baseline CD4 count (median 673 cells $/ \mathrm{mm}^{3}$ ), proportion with HIV-1 RNA<20 (77\%) and HIV duration were similar (9yrs). Baseline sTNF-RI and -RII were positively correlated KYN, KYN:TRP, QA and QA:KA (all p<0.01). hsCRP and IL6 were positively correlated with QA and QA: KA. After adjusting for demographics and factors different between groups, depressed participants had higher inflammatory markers with the exception of IL6 and slightly lower tryptophan metabolites than controls (differences not statistically significant). Higher baseline tryptophan, but not other metabolites nor inflammation markers, was associated with higher PHQ over time $(p=0.04)$. Last, none of the markers were associated with depressive symptom remission at 6 or 12 months.

Conclusion In the context of ART-treated HIV, tryptophan metabolites and inflammation markers were strongly correlated, but neither appear to be related to symptoms of depression.

\section{P303 GIANT CONDYLOMATA ACCUMINATA ASSOCIATED WITH HIV AND HEPATITIS CASE REPORT}

${ }^{1} \mathrm{~T}$ Deleva Jovanov, ${ }^{2} \mathrm{~N}$ Teovska Mitrevska*, ${ }^{3} \mathrm{~A}$ Emurlai. ${ }^{1}$ Health Care Unit Dermatology Department, Skopje, Macedonia; ${ }^{2}$ Remedika general hospital Dermatology department, Skopje, Macedonia; ${ }^{3}$ Clinical hospital Tetovo Dermatology Department, Tetovo, Macedonia

10.1136/sextrans-2021-sti.361

Giant condylomata acuminata also known as Buschke-Löwenstein tumor (BLT) is a benign, slow-growing, locally destructive cauliflower-like lesion usually in the genital region. The disease is usually locally aggressive and destructive with a potential for malignant transformation. The causative organism is human papilloma virus. The most common risk factor is immunosuppression with HIV; however, any other cause of immunodeficiency can be a predisposing factor. They are prevalent in human immunodeficiency virus (HIV)-infected individuals and sexually active populations at risk for HIV acquisition. Some reports have suggested that immunosuppression is a risk factor for recurrence of a condyloma. Thus, we observed the association for a giant anal condyloma in human immunodeficiency virus (HIV)-positive patient and patient with hepatitis C.

Sexually transmitted diseases (STDs) showed a higher prevalence of hepatitis B, followed by hepatitis C, syphilis and HSV.

We present 2 patients male who practice sex with males and have giant condyloma in anal region. For every patient who is entering at operating room we do the screening for STD and hepatitis and we found out that in these two patients who have a giant condulomata accuminata in the anal region were positive on HIV and hepatits C.

We performed laser Co2 1064nm excision of the lesion and local cytostatic imuqimod after the threat with laser and send the patients in STD department for the therapy and further investigation regarding health condition.

On the basis of our work and literature, it can be concluded that giant anogenital warts occur in almost one-third of the male population infected with HIV and factors associated with a higher risk of being diagnosed with anogenital warts as a co-infection with Hepatitis B or C.

\section{P304 THE IMPACT OF ACTIVE SURVEILLANCE AND THE COVID-19 PANDEMIC ON RECRUITMENT OF RESEARCH PARTICIPANTS WITH EARLY SYPHILIS IN CALI, COLOMBIA}

${ }^{1} N$ Romero Rosas*, 1,2J García, 1,2L Ramírez, 3,4K Hawley, ${ }^{1}$ E López-Medina, 1,3,4,5J Salazar. ${ }^{1}$ Centro Internacional de Entrenamiento e Investigaciones Médicas (CIDEIM), Cali, Colombia, Av. La Maria \#19-225, Cali, Valle Del Cauca, Colombia; ' Universidad ICESI, Cali, Colombia., Cali, Colombia; ${ }^{3}$ Department of Pediatrics, University of Connecticut School of Medicine, Farmington CT, USA., Farmington, USA; ${ }^{4}$ Division of Infectious Diseases, Connecticut Children's, Hartford CT, USA, Hartford, USA; ${ }^{5}$ Department of Immunology, University of Connecticut School of Medicine, Farmington CT, USA., Farmington, USA

\subsection{6/sextrans-2021-sti.362}

Background Syphilis remains as a global public health threat. Understanding the immune response and the molecular variability of $\mathrm{Tp}$ is needed for vaccine development. However, most clinical studies do not reach their target sample size. The aim of this study was to describe our experience recruiting early syphilis cases for translational studies in Cali, Colombia.

Methods The monthly number of enrolled participants (MNEP) was obtained from medical records. Three distinct periods were identified. During period 1 (July-2003 to December-2018) the study personnel visited the staff of 87 health institutions and presented the eligibility criteria. During period 2 (May-2019 to March-2020), in addition to staff visits, the active surveillance (AS) of syphilis test results was conducted and an HIV outpatient clinic (HOC) was added to the network. During period 3 (March-2020 to January-2021) the COVID-19 pandemic reached Colombia. The median and interquartile range (IQR) of the MNEP was calculated. The frequency and proportion of subjects from HIV and AS was calculated for periods 2 and 3. Finally, to assess the association between the proportion of months with $0,1,2,3$ or 6 participants and the period the Fisher's exact test was used. Results 159 months were included in the analysis. The median MNEP was 1 for the three periods, while the IQR was $0-1$, 